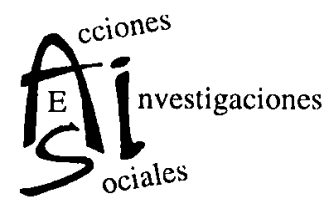

\title{
La regulación territorial de las actividades recreativas en los documentos de planificación ambiental de los parques naturales andaluces de montaña
}

\author{
Ana María LuQue GIL \\ BECARIA DE INVESTIGACIÓN \\ DEPARTAMENTO DE GEOGRAFÍA \\ UNIVERSIDAD DE MÁLAGA
}

El aumento del interés del uso recreativo y turístico en los espacios naturales protegidos ha condicionado la aparición de nuevos conflictos e impactos ambientales, lo cual obliga a llevar a cabo una correcta planificación y regulación del uso público en los mismos.

Las exigencias de sostenibilidad de estos espacios condicionan que sus documentos de planificación deban ordenar y gestionar adecuadamente estas prácticas recreativas, sin embargo, gran parte de los planes de ordenación y gestión carecen actualmente de una regulación óptima de dichas actividades desde un punto de vista territorial.

En este artículo se va a proceder a analizar la regulación territorial de las prácticas recreativas en los documentos de planificación ambiental, escogiéndose, a modo de ejemplo, una serie de espacios naturales 
protegidos ubicados en la región andaluza: los Parques Naturales andaluces de montaña.

Palabras clave: planificación ambiental, uso público, actividades recreativas, Parques Naturales, sostenibilidad ambiental, ordenación territorial. 


\section{La regulación territorial de las activida- des recreativas en los documentos de planificación ambiental de los parques naturales andaluces de montaña}

Ana María Luque Gil

\section{Introducción}

En la actualidad se asiste a una creciente valoración de los espacios naturales de elevada calidad ambiental, la mayor parte de los cuales cuentan con alguna figura de protección legal. Este interés se percibe en el incremento del número de visitantes a estos espacios y en la aparición de nuevos usos y aprovechamientos, entre los que se encuentran los recreativos y deportivos.

Esta utilización creciente, unida a la fragilidad ambiental de los espacios, puede llevar a un fenómeno de masificación que afecte gravemente a la conservación de estos enclaves de elevado interés natural, lo cual ha supuesto la aparición de una normativa que pretende asegurar el correcto aprovechamiento recreativo de estas áreas.

La legislación deportiva, turística y ambiental suelen presentar escuetas referencias en relación con las prácticas recreativas en el medio natural no entrando las mismas, por lo general, en consideraciones relacionadas con la ordenación espacial de éstas actividades. Por el contrario, la mayor parte de las normas que establecen algunas indicaciones territoriales para la práctica de estas actividades en el medio natural andaluz son las vinculadas con espacios naturales protegidos debido a que, al demandar la mayoría de estas prácticas recursos naturales de calidad, se suelen ubicar en espacios que cuentan con alguna figura de protección ambiental.Los documentos de planificación principales de estos espacios son los PORN (Planes de Ordenación de los Recursos Naturales) y los PRUG (Planes 
Rectores de Uso y Gestión), por lo que su consulta resulta obligatoria en el estudio de la regulación de usos o actividades en estas áreas. Es en estos documentos donde se recogen el mayor número de limitaciones y sanciones con relación a la práctica recreativo-deportiva en la naturaleza.

En este artículo se va a proceder a analizar la regulación territorial de estas prácticas recreativas dentro de los documentos de planificación ambiental de los Parques Naturales andaluces de montaña. Se han seleccionado dichas áreas porque en las mismas es donde se da un compromiso más difícil entre conservación y uso recreativo del medio, y porque es básico que estos espacios cuenten con una adecuada ordenación y gestión de las actividades para evitar el posible deterioro de los mismos derivado de este uso.

\section{Los Parques Naturales andaluces de montaña}

La Red Andaluza de Espacios Naturales Protegidos (RENPA) empieza a configurarse en el año 1929 cuando el "Torcal de Antequera" es declarado Sitio Nacional junto con el "Picacho de la Virgen de la Sierra" en Cabra. Tras esto otras fechas de interés fueron el año 1969, cuando se crea el Parque Nacional de Doñana, y 1984 momento a partir del cual comienzan a realizarse las primeras transferencias en materia medioambiental a nuestra región ${ }^{1}$.

Tras este momento hay que esperar al año 1989 en el cual vio la luz la Ley $2 / 89$, de 18 de julio, de Inventario de los espacios naturales protegidos y de la flora y fauna silvestre. Tal y como indica Mulero (2001) las consecuencias más importantes de esta ley fueron, en primer lugar, el fuerte avance que experimentó la declaración de espacios protegidos en Andalucía, pasando a partir de esta fecha a contar nuestra región con más

\footnotetext{
${ }^{1}$ Paralelamente, empezaron a declararse una serie de Parques Naturales en Andalucía al amparo de la Ley nacional de Espacios Naturales Protegidos del año 1975: Parque Natural de Grazalema (1984), Sierras de Cazorla, Segura y las Villas (1986), Sierra de María-Los Vélez (1987) y Cabo de Gata-Níjar (1987) (Benayas del Álamo, 2000, 44).
} 
del $17 \%$ del territorio bajo alguna figura de protección ambiental (frente al $0.6 \%$ que existía en 1988) y con 81 áreas protegidas frente a las tres existentes en 1970 o a las veintidós de 1988; y, en segundo lugar, la diversificación de las figuras de protección respecto a la situación preautonómica, uniéndose a las categorías de parques, reservas naturales, monumentos naturales y paisajes protegidos tomadas de la ley $4 / 89$, las de paraje natural, reserva natural concertada y parque periurbano.

Esta ampliación experimentada por la superficie protegida ambientalmente en nuestra región, va a continuar a partir de ahora al amparo de la Red Natura 2000, con la cual se pretende "crear una red europea de espacios naturales, integrada por Lugares de Interés Comunitario (LIC). En Andalucía se han incluido 128 LIC con una superficie equivalente al $27.8 \%$ de la superficie autonómica" (Mulero, 2001, 147), con lo cual se prevé que siga aumentando progresivamente la superficie protegida².

A escala internacional la figura de protección de Parque es la más extendida y la que cuenta con mayor aceptación.Del mismo modo, tanto a escala nacional como regional, es la figura de Parque la que ha ido adquiriendo mayor representatividad ya que estos suponen formas de protección blandas y permisivas en comparación con otras figuras de protección.

Uno de los primeros rasgos percibidos en la caracterización geográfica de la red de espacios naturales protegidos de Andalucía es que los espacios naturales de montaña son los de mayor relevancia, según Mulero Mendigorri casi un $90 \%$ de la superficie protegida en nuestra región se ubica en ámbito montañoso, por tanto, "la montaña se ha convertido en el ámbito básico de la expansión protectora de la etapa autonómica" (Mulero, 2001, 147). Por otro lado, el protagonismo adquirido por la figura de Parque Natural dentro de la RENPA es extrapolable al espacio montañoso, donde se da el predominio de la figura de Parque Natural frente a otras figuras de protección dominantes en diferentas áreas geográficas (por ejemplo las Reservas Naturales en áreas de vega y campiña).

${ }^{2}$ Actualmente la red de Espacios Naturales Protegidos de la región andaluza consta de más de 100 espacios que suponen el 19\% del territorio andaluz distinguiéndose, dentro del mismo, dos Parques Nacionales, 24 Parques Naturales, 31 Parajes Naturales, 28 Reservas Naturales, 3 Reservas Naturales Concertadas, 17 Parques Periurbanos, 35 Monumentos Naturales y un Paisaje Protegido. 


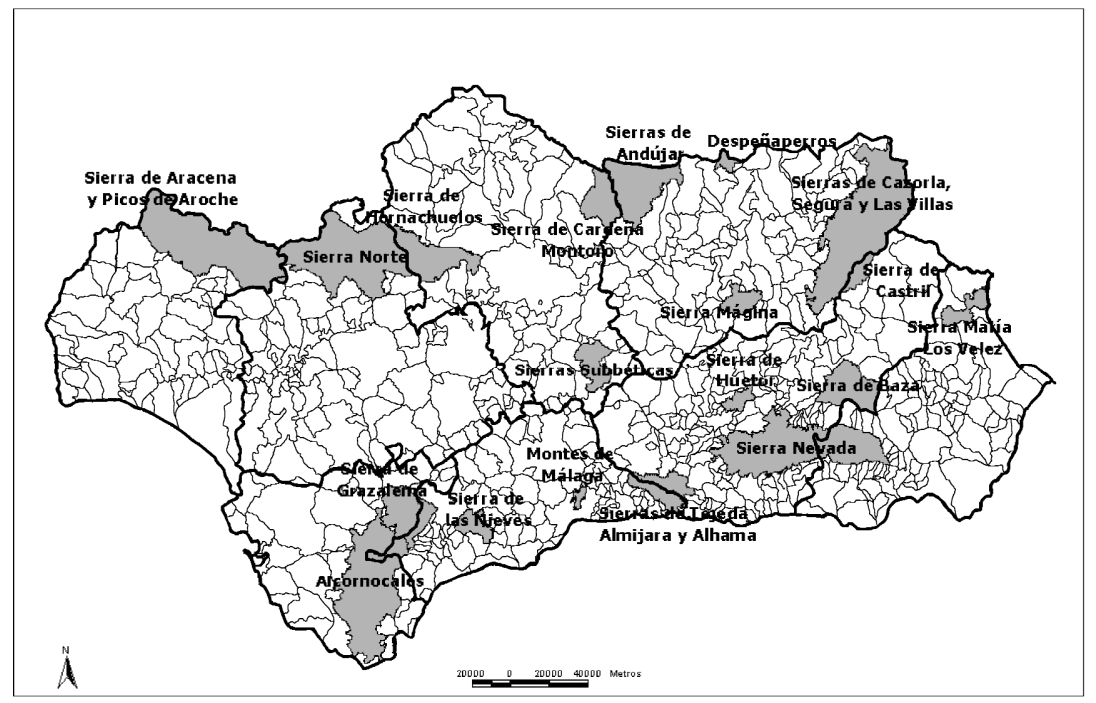

Figura 1.Localización de los Parques Naturales andaluces de montaña.

En consecuencia, el estudio de la interrelación entre ambos aspectos, Parques Naturales y montaña, se plantea a priori bastante atractivo, sobre todo considerando que de los veinticuatro Parques Naturales existentes en nuestra comunidad, diecinueve se ubican en zonas montañosas (Sierra de Aracena y Picos de Aroche, Sierra Norte, Sierra de Grazalema, Alcornocales, Sierras de Cardeña y Montoro, Sierra de Hornachuelos, Sierras Subbéticas, Sierra de las Nieves, Montes de Málaga, Sierras de Tejeda, Almijara y Alhama, Sierra de Huétor, Sierra de Baza, Sierra Nevada, Sierra de Castril, Sierra María-Los Vélez, Sierra de Andújar, Sierra Mágina, Despeñaperros, Sierras de Cazorla, Segura y las Villas).

\section{La regulación territorial de las actividades recreativas en los documentos de planificación ambiental}

Dentro de las actividades a desarrollar en los Parques Naturales andaluces la educación ambiental, el ocio/divulgación y la participación ciudadana adquieren un gran protagonismo, 
de ahí que las actividades recreativas y de uso público adquieran un papel relevante dentro de los mismos. Sin embargo, los documentos de planificación que ordenan y gestionan estos espacios no suelen contemplar adecuadamente la regulación de estas prácticas desde un punto de vista territorial y ambiental.

En Andalucía los Parques Naturales se van a planificar y gestionar en base a tres figuras, los Planes de Ordenación de los Recursos Naturales, los Planes Rectores de Uso y Gestión y los Planes de Desarrollo Sostenible. Tan sólo nos detendremos en el análisis de los PORN y PRUG ya que estos son los que ordenan y regulan los usos dentro de los espacios referidos.

EI PORN es un documento marco básicamente normativo y director para las restantes figuras de planeamiento territorial, ambiental y sectorial. Con lo cual, para llevar a cabo el análisis de la normativa que regula las actividades de uso público en los Parques Naturales andaluces de montaña, se debe partir de estos documentos porque son los que deben señalar la localización e intensidad de los usos y actividades permitidas. Los Planes de Ordenación de los Parques Naturales andaluces tienen semejante estructura:

- Diagnóstico: memoria descriptiva del Plan en la cual se realiza un breve análisis de las características biofísicas, socioeconómicas y paisajísticas del parque.

- Memoria Justificativa: en la cual se hace referencia al alcance del Plan y se justifica la propuesta de ordenación.

- Memoria de Ordenación: el plan en sí que, tras una serie de disposiciones preliminares y generales, establece una serie de normas y directrices relativas a la ordenación de Recursos Naturales, a Planes y Actuaciones Sectoriales y al PRUG y PDI (Plan de Desarrollo Integral).

Sin embargo, estas tres partes del documento que ocupan prácticamente la totalidad del mismo, no hacen en ningún parque prácticamente referencia alguna a la regulación de actividades recreativas en los espacios protegidos. La única referencia se encuentra en el último título de los documentos que es el referido a las "Disposiciones Particulares", donde aparece la propuesta de zonificación del espacio y la regulación de usos prevista. En dicha zonificación se distinguen las zonas de:

- Máximo nivel de protección:"espacios de excepcionales valores naturales, científicos, culturales y paisajísticos, en los que 
prevalecerá la conservación y regeneración de ecosistemas, investigación y educación ambiental" (en muchos de los parques se limitarán un gran número de actividades recreativas).

- Nivel intermedio de protección: "áreas con altos valores ecológicos, científicos, culturales y paisajísticos pero con cierto grado de transformación antrópica. En estas prevalecerán la preservación de valores y el aprovechamiento tradicional de los recursos" (en estas áreas ya se permitirá la práctica de un gran número de actividades recreativas).

- Nivel de protección bajo: zonas en que se busca especialmente el aprovechamiento de los recursos junto con la idea de conservación.

Tras la zonificación se regulan los usos que están o no permitidos en cada una de las áreas, por lo que esta última parte del documento es la que nos va a dar alguna referencia de la regulación de actividades de uso público en los Parques Naturales.

- Por último, el documento se ve acompañado por una Cartografía de Ordenación.

La estructura del PRUG es también semejante en prácticamente todos los espacios. Se inicia con una introducción (que de forma reiterativa sirve de diagnóstico del espacio) donde se plantea nuevamente la zonificación propuesta en el PORN. Tras esto aparece el capítulo dedicado a normativa en el que se van a recoger las normas generales y las relativas al uso y gestión de los recursos naturales en el espacio.

Van a ser estas "Normas Generales" y, dentro de ellas, las que hacen referencia al uso público (Capítulo III) las que nos van a interesar. En ellas se cita la regulación de actividades recreativas dentro del espacio analizado.

Nuestro análisis se circunscribirá prácticamente a las indicaciones que se establezcan en este capítulo, este es prácticamente el único apartado donde se hace mención expresa a la regulación de las actividades recreativas. No obstante, dentro del mismo no nos detendremos en las referencias a los servicios de guías en los Parques o a la gestión de los servicios de uso público, sino que sólo se analizarán aquellas secciones y artículos que hagan referencia a las "actividades de uso público".

EI PRUG en los Parques Naturales debe constar de los Programas básicos de actuación para llevar a cabo las indica- 
ciones establecidas. Estos programas incluyen entre ellos los de uso público que son aquellos que "regularán y programarán el desarrollo de actividades recreativas, didácticas, medioambientales, culturales y educativas dentro del Parque Natural". La finalidad básica de este documento es ordenar y programar las actividades recreativas, turísticas y educativas, por tanto, debería ser uno de los textos básicos a analizar, sin embargo, esto no es posible debido a que hasta hace pocos meses sólo el Parque Natural de Grazalema contaba con el mismo y, por tanto, es el único que se estudiará en este trabajo. En el resto de los casos debemos conformarnos con las breves consideraciones que sobre la regulación de dichas actividades aparece en los PORN y PRUG de los Parques.

El contar con una normativa adecuada es fundamental para que, desde un punto de vista espacial, el aprovechamiento recreativo de los Parques Naturales sea el correcto, evitándose de este modo un mal uso del territorio bien porque la regulación sea demasiado permisiva (surgiendo problemas de impactos ambientales), o bien porque la misma resulte excesivamente restrictiva (limitándose con ello el derecho de la población al uso y disfrute de los espacios protegidos).

\subsection{El tratamiento de las actividades recreativas en los PORN y PRUG de los Parques Naturales andaluces de montaña}

A continuación vamos a proceder a analizar los PORN y PRUG de los Parques Naturales andaluces de montaña para comprobar como se han regulado estas prácticas recreativas, desde un punto de vista territorial, hasta principios del año $2003^{3}$.

${ }^{3}$ A lo largo del año 2003 un gran número de Parques han procedido a revisar sus documentos de planificación ambiental. Los Parques de Montes de Málaga, Sierra de Baza, Sierra de Huétor, Cardeña y Montoro, Sierra de Aracena, Sierra de las Nieves y Hornachuelos ya han aprobado sus respectivos decretos por los que cuentan con un nuevo Plan, no obstante, el análisis realizado se circunscribe al año 2002, de ahí que en el caso de estos espacios se haga referencia a los anteriores documentos de planificación y no a los que se encuentra en vigencia actualmente. 


\section{Parque Natural Sierra de las Nieves}

En el PORN de este Parque aparecen escasas referencias en relación con la regulación de actividades recreativas, además de que en muchos casos estas indicaciones son enormemente aleatorias y dejan las decisiones en manos de la "conciencia ambiental" del planificador. Esto se percibe, por ejemplo, en el Capítulo V del Título III que hace mención a la "Ordenación de los Recursos Forestales", aquí en el artículo 62.2 se establece que: "por razones de protección o conservación, en zonas o caminos forestales de los montes públicos, pueden establecerse limitaciones al tránsito de personas, animales y vehículos que podrán contemplar la prohibición total o restricciones de los mismos, tanto temporales como permanentes".

Sí se hace expresa referencia en los artículos 144 y 145 dentro del título IV de "Normas y Directrices relativas a Planes y Actuaciones Sectoriales", a la "prohibición de utilizar armas de fuego con fines no cinegéticos o deportivos y a la prohibición de la instalación de campos de tiro", estableciendo el Plan la primera limitación a la práctica de actividades recreativas en este Parque Natural (prohibición de instalar campos de tiro en cualquier de sus modalidades: con arco, con carabina o al plato).

El documento también establece una zonificación de usos según las diferentes áreas de protección, en dicha zonificación con relación a las actividades recreativas se indica: por un lado, que en las subzonas de especial interés (A1) "queda prohibida la caza menos en las áreas de la Reserva Nacional de Caza y zonas autorizadas por el AMA" (de nuevo se encuentra cierta ambigüedad en la afirmación, ¿cuáles serán consideradas zonas autorizadas y cuáles no?), y por otro lado, se "limita la entrada a las simas a personal investigador y federado (por medio de un número controlado por el AMA)". En el resto de las áreas se apunta que las actuaciones recreativas deben adaptarse a lo regulado por el PRUG y por el Programa de Uso Público.

Dentro del PRUG el Capítulo III es el que se refiere a la Normativa de uso público, por tanto, es el que básicamente nos va a interesar. En este caso son los artículos 24, 25 y 26 ("De las actividades de uso público") los que hacen mención en cierta medida a la regulación de actividades:

- El Art.24 indica como debe realizarse el tránsito rodado dentro del parque (artículo que aparecerá con semejante redac- 
ción en otros muchos parques): "el tránsito rodado se realizará exclusivamente por pistas, caminos y rutas preparadas al efecto, limitada su velocidad a $30 \mathrm{~km} / \mathrm{h}$, de forma individual o en grupos de menos de 10 y con una separación de más de 200 metros".Con esto se establece la prohibición de ir con vehículos rodados campo a través dentro del parque y se limita el uso de estos a los carriles, caminos y sendas establecidas para tal efecto.

- El Art. 25 hace referencia a la prohibición de realizar vuelos a motor en el parque a una altura inferior a 1.000 m., por tanto, por debajo de esta altura queda prohibida la realización de actividades de vuelo con motor (paramotor, rutas aeroterrestres, ...).

Junto con estas indicaciones que se incluyen en la Normativa sobre uso público, aparece una mención dentro del Título II de "Normas relativas al Uso y Gestión de los Recursos Naturales" que puede tener cierto interés con relación a talleres de naturaleza relacionados con la recolección de hierbas aromáticas y setas:"los aprovechamiento de hierbas aromáticas se realizarán fuera de las subzonas de especial interés y los de setas según los esquemas tradicionales", por tanto, se prohíben los aprovechamientos en la zona $A 1$ y se permite la recolección de setas siempre que se haga de modo tradicional.

Por último, en la normativa particular establecida por zonas (Título III), se hace referencia a la capacidad que tiene la Consejería de Medio Ambiente de limitar el número de visitantes a esta área de especial interés y de señalar las rutas a seguir.

En resumen, las figuras que planifican este espacio natural sólo dan breves apuntes en relación con la regulación de actividades recreativas en el espacio, además de que muchas de estas referencias son bastante ambiguas y dejan abierta la posibilidad de modificar lo establecido por parte del gestor del espacio natural, por ejemplo, la posibilidad de establecer limitaciones al tránsito de personas y vehículos en algunos caminos forestales o la de limitar la entrada a cuevas y simas. Sólo se hace referencia clara a las prohibiciones de las actividades de tiro y de vuelo con motor a menos de $1.000 \mathrm{~m}$. en todo el parque y de cazar en las áreas de protección $\mathrm{A} 1$; el resto son limitaciones que puede establecer, si lo considera pertinente, el Organismo Ambiental a determinadas actividades como son: 
rutas en vehículos $4 \times 4$, espeleología y aprovechamiento de setas e hierbas aromáticas.

\section{Parque Natural Montes de Málaga}

Muy poco se indica en el PORN de este Parque con relación a la regulación de actividades. Dentro de la zonificación del mismo se establecen tan sólo dos niveles de protección (B y C) y la referencia a actividades en cada una de las mismas es bastante escueta:

- Subzonas B1 (de especial protección): "limitación de caza a casos especiales de control de poblaciones y en prevención de daños mayores que se pudieran producir sobre vegetación o fauna".

- Subzonas C1 (de recreo en general): "sólo se permitirá el recreo concentrado en las áreas establecidas al efecto"; "la AMA podrá cerrar o modificar los itinerarios turísticos"; "la acampada o pernocta se adaptará a lo dispuesto por el AMA, exigirá un permiso para realizar estas actividades"; "la renovación del campo de tiro "el francés" requerirá un informe por parte del AMA".

Aquí tan sólo se recogen ciertas limitaciones que puede llegar a establecer el Organismo Ambiental a actividades (recreo concentrado, senderismo, acampada y tiro) en casos concretos, de nuevo aparece una gran ambigüedad en los conceptos planteados. Como tal no aparece prohibida ninguna actividad dentro del documento.

Dentro del Plan Rector de Uso y Gestión, el Capítulo III que hace referencia a la Normativa de uso público, es el que da alguna información sobre la regulación de actividades (artículos 24 al 31):

- Art.24: establece que la acampada o pernocta se adaptará a lo dispuesto por el organismo ambiental, por tanto, se limita a hacer referencia a la normativa sobre acampada existente y no hace ninguna concreción de aquellas zonas del parque donde se puede o no acampar, donde se prohíbe según determinadas condiciones, etc.

- Art. 25: Indica que el tránsito de personas y vehículos se realizará por las zonas establecidas para cada caso, sin embargo al no concretar cuáles son estas zonas, puede ser que se refiera a la prohibición de recorrer campo a través el parque debiéndose utilizar los caminos y rutas convenientemente trazados. 
La ambigüedad es menor en el caso de la "prohibición de pruebas deportivas de vehículos a motor en los caminos forestales del parque", sin embargo, sí se van a permitir (de nuevo con permiso del Organismo Ambiental) la celebración de estas pruebas en la carretera que circunda el espacio natural.

Art.26: establece las mismas limitaciones al tránsito de vehículos rodados que el Parque Natural Sierra de las Nieves.

Art.27: indica la prohibición de vuelos a menos de $1.000 \mathrm{~m}$ de igual modo que lo encontrábamos en el PRUG de Sierra de las Nieves.

Los artículos 28 al 31, ya no hacen referencia concreta a actividades en sí. Indican el equipamiento mínimo que deben tener las áreas recreativas, la prohibición de encender fuego fuera de las zonas establecidas para ello, etc.

Al igual que en el caso de Sierra de las Nieves, se hace referencia a la limitación en el aprovechamiento de plantas aromáticas, que en este caso "se realizará fuera de las zonas de especial protección" (art.42), al igual que la obligación del Organismo Ambiental en este mismo espacio (B1), de señalar las rutas y el número de visitantes permitidos.

De nuevo la regulación de actividades recreativas es prácticamente inexistente y las indicaciones que aparecen muy ambiguas. Tan sólo aparecen prohibiciones expresas a la realización de pruebas deportivas a motor en el interior del parque, a la práctica de vuelo a motor a menos de $1.000 \mathrm{~m}$. y al aprovechamiento de plantas aromáticas en subzonas B1, en cambio, la actitud con el resto de actividades realizadas o potenciales de practicar es mucho más permisiva: caza, senderismo, rutas en BTT, recreo concentrado (en zonas habilitadas para tal fin), acampada o tiro (campo de tiro habilitado para ello).

\section{Parque Natural Sierra de Grazalema}

En el Plan Director de Uso y Protección ${ }^{4}$ las únicas referencias a la regulación de actividades recreativas que aparecen se vinculan con la zonificación general de usos y actividades que se hace en el espacio.

\footnotetext{
${ }^{4}$ El parque carece de PORN y PRUG.
} 
En las zonas de reserva (A), queda prohibida la caza y la circulación de vehículos a motor (coches todo terreno, quads, etc.), permitiéndose otras actividades recreativas como el montañismo o la espeleología.

En el resto de áreas de protección (B y C) todas las actividades turístico-recreativas son compatibles (siempre considerando lo indicado en el Programa de uso público).

Ya en el Plan Director de Uso y Protección se establece que el Programa de uso público debe "regular y promover la realización de actividades culturales, educativas y recreativas en el parque". El Programa de uso público ha surgido por la necesidad que tiene este Parque de regular el mismo debido a la presión que éste genera sobre el espacio.

En el Diagnóstico realizado en el documento, se percibe someramente que áreas deben quedar limitadas al uso público porque son zonas especialmente frágiles por diversas razones: cuevas con hibernación y cría de quirópteros, áreas de concentración de la cabra montés, zonas de nidificación de aves y áreas con peligro de incendio forestal. Frente a estas zonas que no deben contar con uso público, aparecerán otras con uso público sujeto a autorización y con uso público no sujeto a autorización.

La parte del documento que más interés despierta para nuestro fin es aquella que contempla la regulación de las actividades de uso público. Algunas de las normas más interesantes contempladas en este apartado en relación con actividades recreativas son:

- Senderismo y Montañismo: limitado a senderos, caminos públicos o vías pecuarias así establecidas; el Organismo Ambiental podrá limitar el acceso a estos senderos; en "senderos guiados", se limita su entrada a un cupo diario de personas, obligatoriedad de guías, etc. (Garganta Verde, pinsapar o Puerto de los Acebuchales); en "senderos autoguiados", no es necesaria autorización y se pueden recorrer a pie, en bicicleta de montaña o a caballo, no es necesario llevar guía y se localizan fuera del área de reserva; en "senderos especiales", donde se realiza la práctica del montañismo en áreas de reserva, se establece un límite de entrada diario y mensual (Torreón y Crestería).

- Ala delta y Parapente:las prácticas de vuelo se realizarán en el cerro Albarracín (que cuenta con una pista de ala delta), 
aunque se podrán dar permisos excepcionales para la competición en otras zonas; prohibición de vuelo del 1 de enero al 15 de junio en un área establecida; prohibición de volar en zonas de protección máxima $(A)$.

- Espeleología: sólo grupos federados y empresas especializadas pueden realizar la travesía en el sistema Hundidero-Gato.

- Escalada: regulada y permitida la práctica en una serie de enclaves durante todo el año (Cortados de Montejaque y Benaoján, Peñón Grande,...), tan sólo queda limitada en la zona del Saltadero (Ubrique) desde el 1 de julio al 31 de diciembre.

- Actividades náuticas: sólo se permiten embarcaciones sin motor en los embalses de Zahara de la Sierra-El Gastor y en el río Guadiaro.

- Rutas a caballo: permitidas en senderos guiados y autoguiados (siempre en grupos con menos de 12 caballos y dos guías).

- Rutas en vehículos 4 x 4: tan sólo existe una ruta permitida, Puerto Acebuches-Benamahoma (con un límite de seis vehículos por día).

- Acampada: limitada a las áreas de acampada establecidas para tal, aunque la Consejería puede otorgar algún permiso excepcionalmente.

\section{Parque Natural Sierra Tejeda, Almijara y Alhama}

EI PORN del Parque se aprobó por medio del decreto 145/1.999, de 15 de junio y el PRUG aún se encuentra en elaboración.

Las referencias a las actividades recreativas dentro de este documento son muy escasas, sin embargo, se plantea como necesario llevar a cabo una buena regulación ya que, según el PORN, "la riqueza natural y el atractivo paisajístico del parque hacen que este sea un entorno ideal para el disfrute del tiempo de ocio".

Las únicas indicaciones existentes, son aquellas relacionadas con la regulación de actividades realizada tras la zonificación ambiental del espacio. De este modo en las áreas de máxima protección (zonas A) se indica la compatibilidad en la misma de la práctica de actividades como la caza, pesca, actividades didácticas o actividades recreativas de bajo impacto (sin embargo, no se indica cuales son consideradas actividades de este tipo, apuntándose para ello a lo establecido en la planifica- 
ción de uso público que, como en la mayor parte de los espacios protegidos andaluces, es claramente inexistente).

Las únicas prohibiciones expresas a prácticas recreativas en este espacio son: la circulación de vehículos a motor y las actividades recreativas y deportivas que impliquen la instalación de una infraestructura permanente (por ejemplo picaderos, etc.) 0 aquellas que superen la afluencia de visitas por encima de la capacidad de acogida (necesitándose para ello estudios de este tipo que indiquen cual es dicha capacidad).

En las zonas de protección $\mathrm{B}$, también están prohibidas las actividades recreativas motorizadas (rutas en vehículos todo terreno, quads, motocross, etc.).

Por tanto, referencias muy breves y tan sólo claras en la incompatibilidad de las prácticas con vehículos en las zonas de protección A y B.

\section{Parque Natural Alcornocales}

Las primeras indicaciones con respecto al uso público y la práctica de actividades recreativas en el parque, la encontramos en la regulación de usos que se realiza tras el proceso de zonificación de este espacio protegido. Prácticamente no aparecen referencias concretas a la regulación de las actividades recreativas en las distintas áreas, tan sólo se dan una serie de ideas genéricas: "en las zonas A1 estarán prohibidos todos los aprovechamientos, menos los necesarios para la mejora de los valores naturales y ecológicos de las mismas, por lo que se establecerán estrictos controles de acceso" (art. 15.1.).

Por tanto, se sobreentiende que todas las actividades recreativas y de uso público están limitadas en esta zona A1, a no ser que el Organismo Ambiental las considere necesarias para "mejorar los valores naturales y ecológicos de las mismas" (de nuevo la ambigüedad aparece y el artículo se presta a diferentes interpretaciones).

Lo escueto y poco explícito que es el PORN en este sentido, no se acompaña de un PRUG de semejantes características, de este modo en el apartado de Normativa del documento y concretamente en el referido a Normas de uso público, aparecen una serie de referencias a las actividades de uso público (artículos 27 al 29), en las cuales se establecen algunas limitaciones territoriales a las actividades: 
- Art.27: regula las características que deben cumplir aquellas organizaciones o personas que desarrollen actividades en el Parque:"Acceso libre a montes públicos menos en zonas de protección $\mathrm{A}$ donde el acceso será limitado y hará falta autorización; Necesidad de llevar documentación ordinaria y licencia federativa los miembros de grupos de montañismo, espeleología y caza; Posibilidad de expedir permisos a grupos por una duración de un año y prorrogable; Las personas no federadas necesitan una autorización expresa cada vez que realicen la actividad;Regulación de la espeleología en el complejo "Ramblazo-Motillas".

Desde un punto de vista territorial, tan sólo aparecen limitadas las actividades en la zona A (se necesita autorización) y la espeleología en el complejo mencionado, las demás apreciaciones sólo hacen referencia a la documentación y permisos que se deben llevar para practicar la actividad (artículos semejantes aparecen en otros muchos parques).

- Art.28: este artículo ya es un tanto más preciso en cuanto a la regulación de actividades:

- Se prohíbe la acampada fuera de las zonas establecidas al respecto (aunque de nuevo se deja libre la concesión de permisos extraordinarios por parte de la Consejería).

- Posibilidad de limitar o cerrar incluso el tránsito de visitantes a determinados espacios por parte de la Consejería (cuando lo aconseje la conservación del mismo).

- Si algunas zonas son adecuadas para practicar escalada, parapente, ala delta o ultraligeros, se estará a lo dispuesto en los Programas Básicos de Actuación (Programa de Uso Público), esta es una apreciación sin ninguna traducción territorial, es decir, no se indica donde se están practicando o donde se pueden practicar o no las actividades, por tanto, al ser enormemente teórica no sirve para nuestros presupuestos.

- Art.28.6: de nuevo se establecen limitaciones a una actividad (en este caso el excursionismo), indicándose simplemente que se establecerán itinerarios fijos (debidamente señalizados) y que el organismo ambiental podrá limitar la actividad según la demanda de los itinerarios, fragilidad de los ecosistemas y época del año. 
De nuevo no aparece ninguna referencia territorial, es decir, no se indica cuáles son los itinerarios con más probabilidades de limitación de usuarios, por lo que no se puede indicar concretamente las áreas en las que potencialmente podría verse impedida la práctica de la actividad.

Otra breve indicación aparece en las Normas Relativas al Uso y Gestión de los Recursos Naturales, aquí se establecen dos prohibiciones concretas:

- Prohibición de las prácticas de: motociclismo, motocross o carreras de vehículos a motor. Al igual que se limita el tránsito de vehículos a motor fuera de las carreteras y caminos públicos, tan sólo permitiéndose los necesarios para que se desempeñen las labores de trabajadores y empleados y los desplazamientos de residentes y visitantes (esta última apreciación condiciona que los visitantes puedan pasar con sus vehículos fuera de los caminos y sendas trazados para tal).

- Prohibición total de circulación con vehículos a motor en el área $\mathrm{A} 1$ (menos los vehículos de la Consejería y autorizados). Como en los planes analizados precedentemente, las referencias territoriales a la regulación de actividades recreativas y de uso público son muy escuetas, apareciendo tan sólo prohibiciones concretas en relación con la práctica del motocross, motociclismo y carreras de vehículos a motor en todo el parque, con la acampada fuera de las áreas establecidas para tal efecto (camping o zonas de acampada libre organizada) y con la circulación de vehículos en el área A1; lo demás son simplemente regulaciones poco estrictas de actividades como espeleología 0 excursionismo, más orientadas a temas de licencias y permisos que a indicaciones territoriales concretas.

\section{Parque Natural Sierra de Huétor}

EI PORN establece tres tipos de zonas ( $A, B$ y $C$ ) de más a menos protección, en ellas se hace cierta mención a la regulación de actividades:

- Zonas de protección A: "serán incompatibles cualquier uso distinto al científico o cinegético", por tanto, aquí estará prohibida cualquier actividad recreativa que no sea la cinegética.

- Zonas de protección B: además de las consideraciones anteriores ya serán compatibles en las zonas B1 "el senderismo y la circulación de vehículos por pistas forestales"; mientras que en las zonas B2 será incompatible el uso público en las zonas 
sometidas a trabajos forestales; en las áreas B3 será incompatible el uso público; y en la B4 será compatible el uso público de los excursionistas que accedan a pie e incompatible el acceso de vehículos a motor; en la zona B5 ya serán compatibles prácticamente todas las actividades de uso público.

En la sección segunda del Capítulo III del PRUG aparecen una serie de artículos de interés (art. 24 al 26):

- Regulación de las licencias y permisos que deben portar los practicantes de determinadas actividades (artículo semejante al que aparece en la mayor parte de los PRUG de los Parques).

- Prohibición de acampar o pernoctar fuera de las áreas destinadas para ello.

- Prohibición de circular fuera de las pistas autorizadas para ello con vehículos a motor de cualquier tipo.

- Prohibido la circulación de BTT y caballerías fuera de las pistas o caminos libres.

- Prohibición de las prácticas deportivas de espeleología, escalada y montañismo sin autorización de la Administración.

\section{Parque Natural Sierra de Castril}

Algunas de las indicaciones relacionadas con la regulación de actividades recreativas que aparecen en este PORN son bastante indirectas, por ejemplo, la que establece que en las zonas de máxima protección ambiental (A) "se prohíbe el acceso de cualquier persona ajena a la gestión del parque", lo cual indicaría que se prohibirían en este ámbito todas las actividades de uso público. En las otras zonas de protección B y C, no se hace ninguna indicación específica.

Sí existen referencias más concretas en este sentido en el PRUG, aquí en la sección segunda del Capítulo III que hace referencia a actividades de uso público, encontramos indicaciones en los artículos 24 al 29:

- Art.24: indica que "sólo será posible la acampada en las áreas habilitadas a tal fin", por tanto, se prohíbe esta actividad en todo el parque menos en las zonas que se habiliten para ello.

- Art.27: se refiere a las actividades de escalada y espeleología y en él se dan indicaciones muy generales del tipo: pro- 
hibición de prácticas en las zonas de grado A, posibilidad de contar con guía propio, obligación de portar licencias federativas, etc., sin embargo, no aparece ninguna apreciación de tipo territorial, excluyéndose la limitación posible de practicar ambas actividades en las zonas de grado $A$.

- Art.28: indica que "las actividades aeronáuticas con carácter recreativo se prohíben genéricamente, autorizándose sólo las relacionadas con la gestión del parque y la investigación".

- Art.29: se refiere a las actividades náuticas a practicar en el embalse del Portillo (río Castril). Aquí queda prohibido el uso de embarcaciones a motor con uso deportivo o turístico (por ejemplo, motonáutica, etc.), siendo únicamente libre la práctica de vela o piragüismo $u$ otros deportes sin motor (actividades que también podrán quedar restringidas en épocas del año o zonas del embalse).

Las referencias a la regulación de actividades en estos documentos de planificación son muy escuetas tan sólo: prohibición de practicar actividades en las zonas de protección A, prohibición de acampar fuera de las áreas establecidas para tal fin, prohibición de las prácticas aeronáuticas dentro del parque y prohibición de las prácticas náuticas a motor.

\section{Parque Natural Sierra de Baza}

Desde un punto de vista territorial, ya en el PORN aparecen algunas indicaciones con relación a la regulación de actividades de uso público:

- Zonas de protección A1, prohibición de cualquier uso distinto del científico (quedando incluidas en la prohibición las prácticas recreativas).

- Zonas de protección A2, será incompatible la "circulación de vehículos por las pistas forestales en los montes públicos (menos los autorizados por la Administración) y el acceso masivo de visitantes a estos territorios" (esta segunda referencia utiliza el concepto masivo sin indicar que número de personas o visitantes se entienden como tal).

En las zonas de protección $\mathrm{B}$ y $\mathrm{C}$, no se expresa incompatibilidad manifiesta ante las actividades recreativas.

EI PRUG se refiere también escasamente a la regulación territorial de las actividades recreativas y así, sólo aparece una 
prohibición expresa a la práctica de acampar fuera de las áreas habilitadas para ello.

Junto a esto, en el artículo 25 de dicho Plan, también aparecen una serie de indicaciones sobre las licencias y pautas a seguir por los practicantes de escalada y espeleología dentro del Parque, no obstante, la regulación territorial es prácticamente inexistente.

\section{Parque Natural Sierra Nevada}

Escasas referencias aparecen en el PORN con relación a la regulación territorial de actividades recreativas, así por ejemplo en la Subzona de Protección A1 "Altas Cumbres Occidentales", se prohíben específicamente las instalaciones deportivas de esquí alpino; en la subzona A6 "Áreas del Trevenque", se prohíbe la circulación motorizada por la pista forestal de la Cortijuela (fuera de los horarios establecidos) y las actividades deportivas o de ocio que requieran motorización; subzona de protección A7 "Montenegro" no se considerarán compatibles las actividades deportivas motorizadas; subzona A8 "Humedales y turberas de Padul" son incompatibles las actividades deportivas o de ocio con vehículos a motor y la caza y pesca en todas sus manifestaciones.

En estos artículos se hace mención expresa a alguna actividad recreativa, sin embargo, en otros muchos, por ejemplo, los relacionados con nuevas construcciones, actuaciones impactantes, etc., pueden tener alguna cabida.

En el PRUG, en el capítulo segundo se analizan las infraestructuras de uso público, estableciéndose una serie de actuaciones prioritarias a realizar:centros de acogida y recepción; señalización, adaptación y mejora de los circuitos, creación de aulas de la naturaleza, etc. (aunque terntorialmente no da mucha información de que zonas son prioritarias o restrictivas para su localización).

En la sección tercera del mismo capítulo, aparecen las referencias a las actividades de uso público, de los artículos 29 al 36 nos interesan sólo una serie de medidas:

- Sólo se podrá circular en el Parque Natural por sendas y caminos autorizados para ello.

- La acampada deberá ser autorizada por la Administración.

- Prohibición de vuelo sin motor entre el 1 de enero y el 1 de junio en las áreas de nidificación de rapaces. 
- Prohibición de vuelo con motor en todo el parque.

- Prohibición de la práctica de actividades deportivas con vehículos fuera de los lugares acondicionados (aquí cabría preguntarse cuáles son estos lugares, ya que no se indica nada en este sentido).

\section{Parque Natural Sierra María-Los Vélez}

La regulación de actividades que se realiza tras el proceso de zonificación dentro del Parque y que encontramos en el PORN es muy escueta y ambigua en relación con el uso recreativo, así en la zona de máxima protección (A): se prohíbe la actividad cinegética (menos en el caso de que ésta quede regulada por un Plan técnico de caza) y la instalación de campamentos y prácticas deportivas que no se encuentren reflejadas en el Programa de uso público (inexistente) y reguladas en el PRUG.

Considerando únicamente este documento se puede afirmar que existe una prohibición total en esta zona $\mathrm{A}$ a la ubicación de campamentos. En las zonas de protección $\mathrm{B}$ y $\mathrm{C}$ prácticamente no se hace mención a este tipo de actividades recreativas.

El Capítulo III del PRUG es el que hace referencia a la Normativa de uso público y desde el artículo 24 al 48 se analizan las actividades de uso público desde un punto de vista normativo. De esta serie de artículos han parecido de interés:

- Los artículos 24 al 27, se refieren a la regulación de actividades de tránsito por la red viaria dentro del parque. Los recorridos en el mismo se harán utilizando dicha red y los vehículos de tracción mecánica sólo podrán transitar por "carreteras, caminos vecinales y pistas forestales de libre acceso". A pie y, excepcionalmente en BTT, se podrán transitar además de las anteriores por sendas y veredas señalizadas de "libre acceso".

Todo este tránsito por la red viaria (con las limitaciones indicadas) será libre, pudiendo la Administración establecer limitaciones en determinadas áreas.

- Art.28: "quedan prohibidas todas aquellas actividades en las que se usen armas de fuego a excepción de la actividad cinegética".

- Art.32 y 33: establecen que "la acampada se permitirá en los espacios delimitados a tal efecto como campings públicos y áreas de acampada", prohibiéndose la acampada libre. 
- Art.37, recogen las pautas a seguir dentro del parque por los practicantes de espeleología y montañismo (artículo semejante al de otros muchos parques).

- Art.40: "las actividades aeronáuticas deportivas, podrán ser limitadas en determinadas zonas por la Administración y quedan completamente prohibidas entre el 1 de enero y el 1 de junio en las áreas de nidificación de las rapaces".

Desde el punto de vista de las prácticas recreativas la regulación es tan sólo clara para una serie de actividades (acampada libre e instalación de campamentos, actividad cinegética, actividad de tiro y vuelo), no obstante, eludiendo las prohibiciones específicas a determinadas prácticas, el resto de consideraciones son meramente orientativas y no hacen referencia alguna desde un punto de vista territorial. La regulación de las actividades que aquí aparece es demasiado genérica y prácticamente no se detiene en detalles de interés.

\section{Parque Natural Sierra de Cazorla, Segura y las Villas}

EI PORN establece cinco zonas diferentes dentro del Parque según el interés ecológico que presenten (Zonas $\mathrm{A}$ de máxima protección y E de mínima protección), en cada una de ellas aparece una regulación de usos. De los epígrafes analizados, tan sólo han interesado las siguientes indicaciones:

- Prohibición de acampada y pernoctación en zonas de protección A.

- Prohibición de vuelos con ultraligeros, ala delta y otros medios aéreos en zonas de protección $A$.

EI PRUG también recoge algunas referencias territoriales con respecto a la regulación de actividades de uso público (art. 26 al 31):

- Libre utilización de embarcaciones a vela, piragüismo y otros deportes con motor eléctrico y prohibición del uso de otras embarcaciones a motor.

- Prohibición del montañismo y escalada en los cantiles que sirvan como áreas de nidificación de las rapaces.

- Prohibición de las prácticas aeronáuticas en áreas de reproducción de rapaces entre el 1 de enero y el 1 de septiembre.

- Prohibición del baño en los embalses de Aguascebas, Valdeazores, Aguas Negras y tramos acotados y vedados con trucha común. 
En el capítulo III de dicho Plan, se establecen además las "Normas para la construcción y localización de equipamientos turísticos y de uso público".

\section{Parque Natural Sierra Mágina}

Las referencias territoriales son escasas en las figuras de planificación con respecto a la regulación de actividades. En el PORN tras establecer la zonificación del parque partiendo de criterios ambientales, se indica simplemente que en las zonas $A$ "deberán primar las actividades de investigación e interpretación, también algunas de uso público", por tanto, no se establece ninguna limitación.

EI PRUG también es bastante impreciso y ambiguo en este sentido, los artículos del 24 al 28 de la sección segunda del Capítulo III, son los que hacen referencia a las actividades de uso público, pero con una prácticamente inexistente regulación territorial:

- Art.24:indica las licencias y permisos que deberán portar los practicantes de determinadas actividades.

- Art.25: recoge que si algunas zonas del parque reúnen buenas condiciones para la escalada, parapente, ala delta 0 ultraligeros, se estará a lo dispuesto en los programas básicos de actuación (sin embargo, el espacio no posee aún programa de uso público que pueda regular esto).

La Administración tiene la libertad de limitar el excursionismo o cualquier otra actividad dentro del parque cuando la fragilidad de los ecosistemas así lo indiquen (art.26).

- Art.28: prohíbe la acampada fuera de las zonas establecidas para ello y promoverá dicha actividad en la periferia del parque.

Junto a estas breves referencias, en el capítulo décimo "de protección del espacio de actividades molestas y peligrosas", se establece (art.63) la prohibición del motociclismo, motocross y carreras de vehículos a motor en todo el parque, a la vez que se prohíbe igualmente la circulación de vehículos a motor en la zona de protección A.

\section{Parque Natural Sierras Subbéticas}

En el PORN aparecen escasas referencias sobre regulación territorial de actividades recreativas, sin embargo, se prevé su 
regulación en prevención de posibles deterioros que puedan provocar estos usos. Así, por ejemplo en la zona de protección B se "potenciarán actividades de montañismo, senderismo, paisajismo e interpretación de la naturaleza y se establecerán medidas especiales de protección para conservar cuevas y cavidades (especialmente en la cueva de los murciélagos), etc.".

Las indicaciones del PRUG en este sentido también son escasas, así en las Disposiciones Particulares que se hacen eco de la zonificación, se indica que en las zonas de protección B "se regulará el acceso de visitantes a determinadas áreas para evitar la degradación ambiental y fundamentalmente se establecerán controles en los extremos del recorrido de la Sierra de Zuheros a S ${ }^{\mathrm{a}}$ Cabra".

En ambos documentos no se expresa con claridad como deben quedar ordenadas territorialmente las actividades ni cual debe ser su ubicación prioritaria e idónea.

\section{Parque Natural Sierra de Andújar}

En el Plan de Ordenación aparecen en el capítulo dedicado a la regulación de usos en las diferentes zonas de protección, algunas indicaciones con respecto a actividades de recreo:

- Prohibición de recolección de especies vegetales aromáticas en las zonas de protección A.

- Incompatibilidad de actividades de educación ambiental en las subáreas de alto valor ecológico en las zonas de protección A.

En el PRUG también aparecen una serie de artículos (23-29) en la sección segunda del capítulo III sobre normativa de uso público. En éste lo referido a actividades recreativas que nos interesa:

- No estará permitida la acampada fuera de las zonas establecidas al efecto.

- En el área recreativa del Jándula se establecerán zonas de baño donde estará prohibida la pesca deportiva.

- Prohibición del uso de embarcaciones a motor con potencia superior a $20 \mathrm{HP}$ en los embalses del Jándula y Encinarejo y las de potencia inferior necesitarán permiso.

En el capítulo dedicado a la protección del espacio de actividades peligrosas y molestas, se prohíbe además la circulación de vehículos en las zonas de protección A y la práctica del moto- 
ciclismo, motocross y carreras de vehículos a motor en todo el parque.

Junto a esta serie de referencias aparecen otras bastante ambiguas que dejan abierta la práctica de actividades como escalada, ala delta, parapente o ultraligeros, según lo que se indique en el programa de uso público (del cual carece el parque).

\section{Parque Natural Sierras de Cardeña y Montoro}

En el PORN, tras la zonificación realizada según los estados de los diferentes ecosistemas en el Parque Natural, se hace una regulación de actividades. Dentro de la misma, la única referencia de interés para este trabajo, es aquella que establece la "incompatibilidad de las actividades de montañismo, espeleología, senderismo o acampada en la zona de protección grado A". Sin embargo, de nuevo queda abierta la posibilidad de que la Administración modifique esta limitación ya que "podrá autorizarlas con carácter excepcional”.

Más numerosas son las indicaciones relacionadas con actividades recreativas que aparecen en el PRUG. Los artículos 24 al 29 de la sección segunda del Capítulo III de dicho Plan recogen algunas referencias de interés:

- Art.24: documentación que deben portar los practicantes federados de la actividad de montañismo, espeleología o caza dentro del Parque y pautas a seguir por los no federados.

- Art.25: "si alguna zona se considera idónea para la práctica de actividades de vuelo (ala delta, parapente o ultraligero), se estará a lo dispuesto en los Programas Básicos de Actuación" (del cual carece el parque). Por tanto, no se precisa nada de que zonas van a ser o no aptas para localizar dichas actividades.

- Art.26: indica que la Administración establecerá itinerarios fijos para la práctica del excursionismo (de nuevo no se precisa que zonas son idóneas o no para localizar dichos itinerarios).

- Art.28: prohibición de acampar fuera de las áreas establecidas para ello.

- Art.29: obliga a que las "instalaciones relacionadas con el deporte náutico necesitan autorización de la Administración al igual que el uso de embarcaciones a motor en el embalse de Yeguas". 
Por tanto, las referencias relacionadas con la regulación de las prácticas recreativas en este parque son muy escasas y prácticamente no ofrecen ninguna indicación territorial, tan sólo una doble consideración tiene tal efecto: la prohibición de la práctica del montañismo, espeleología y senderismo en la zona de protección grado $A$, y la prohibición de acampada libre en todo el Parque fuera de las zonas establecidas para tal fin.

\section{Parque Natural Sierra de Hornachuelos}

EI PORN diferencia, al igual que en la mayoría de los parques, zonas de protección A, B y C, según las condiciones ecológicas.

Dentro de las zonas A1 ("Espacios de especial interés"), "quedan prohibidas las actividades de montañismo, senderismo, acampada o espeleología, excepto lo establecido en el PRUG y en los programas de uso público" (del cual carece el parque), en el resto de áreas siempre que estas actividades sean respetuosas con el medio en el que se localicen, están permitidas.

Las consideraciones que en este sentido aparecen recogidas en el PRUG son muy escuetas y prácticamente sin ninguna indicación territorial, así únicamente se hace mención a la "prohibición de la acampada fuera de las zonas establecidas a tal efecto"y a la "prohibición del vuelo en ala delta, parapente u otro medio aéreo del 1 de enero al 1 de agosto en zonas de nidificación de rapaces" (art. 24 y 29).

Por tanto, las únicas referencias con respecto a la regulación territorial de estas actividades son:

- Prohibición de la práctica del montañismo, senderismo, acampada y espeleología en zonas A1.

- Prohibición de la acampada libre en todo el parque.

- Prohibición de las actividades de vuelo en áreas de nidificación de rapaces entre el 1 de enero y el 1 de agosto.

\section{Parque Natural Sierra Norte}

En el PORN las referencias a la regulación de actividades recreativas son escasas o más bien prácticamente inexistentes. En el documento, dentro de las Disposiciones Particulares, cuando se establece la zonificación del espacio protegido, se dan breves referencias a la compatibilidad, por ejemplo, en la Red de Espacios de Especial interés de "las adecuaciones naturalísticas y usos didácticos o científicos, (...) los usos turístico- 
recreativos sobre edificaciones legales ya existentes (...)", pero referencia concreta a la compatibilidad o incompatibilidad territorial de alguna actividad recreativa no se da.

Por el contrario, las referencias a la ordenación y regulación del uso público en general y de las actividades recreativas en particular, si que son mucho más numerosas en el PRUG.

Especial atención cabe prestar a la sección segunda que dentro del Capítulo III hace referencia a las actividades de uso público en dicho documento (artículos 27 al 36):

- Art.27 y 28: informan sobre los requisitos que deben cumplir las instalaciones de camping: donde se deben localizar preferentemente, que condiciones cumplir (infraestructuras, capacidad máxima de acampada, etc.)

- Art. 29: informa sobre el régimen de acampada. Dicha actividad se debe circunscribir a los lugares establecidos para tal efecto, pero como ocurría en otros parques, el Organismo Ambiental puede dar permisos excepcionales por necesidad de conservación del parque natural.

- Art. 30: establece condicionantes a la práctica del senderismo y los recorridos a caballo. Ambas actividades territorialmente se tendrán que limitar a: "terrenos, caminos o vías de carácter público así como a las vías pecuarias"(si se usan caminos particulares se requerirá la autorización expresa del propietario). Al igual que en otros espacios la Administración tendrá la capacidad de limitar el acceso a estas vías cuando así lo considere necesario.

En la regulación de estas actividades, sí que aparece una apreciación que no se había encontrado hasta ahora en ningún parque y hace referencia a la señalización de estos itinerarios, ya que "estará prohibida la realización de inscripciones o dibujos en los elementos naturales para señalizar los recorridos".

- Art. 31: regula que criterios deben cumplir aquellas personas que pretendan realizar las actividades de escalada y espeleología en el interior del Parque Natural. Es un artículo muy parecido al que aparece en otros parques, en el cual se establece que los miembros federados que realicen estas actividades deberán portar con su correspondiente licencia federativa, contar con una autorización anual para la realización de la actividad y con permisos de los titulares en el caso que la actividad vaya a desarrollarse en terrenos particulares. Aquellas 
personas no federadas necesitarán autorización expresa de la Administración cada vez que pretendan realizar la actividad.

En este artículo se hace especial hincapié en los requisitos que deben cumplir los practicantes de la actividad (licencias, permisos, ...) pero no aparece acompañado por una regulación por zonas de la actividad, es decir, no se indica que áreas son más o menos frágiles para el desarrollo de las actividades y en cuales, por tanto, éstas deben contar con alguna limitación.

- Art.32 y 33: se refieren a la práctica de actividades náuticas o aeronáuticas, no indican absolutamente nada y sólo establecen que ambas actividades podrán ser autorizadas por la Administración.

El resto de artículos no concretan nada más y sólo hacen mención a la necesidad de potenciar las zonas recreativas, el equipamiento de las mismas, etc. Por tanto, la información obtenida es muy escasa y prácticamente no se clarifica nada respecto a la regulación territorial de actividades:

\section{Parque Natural Sierra de Aracena y Picos de Aroche}

Dentro del PORN no se hace ninguna referencia explícita a la regulación de actividades de uso público o actividades recreativas. Tampoco en el PRUG se encuentra ninguna indicación sobre actividades de uso público, por tanto, en este parque y, a pesar de su potencial turístico-recreativo y de que en la actualidad se ofertan empresarialmente actividades, no se hace ninguna alusión a la regulación de actividades de recreo en el mismo, dejándose un vacío jurídico y normativo importante en este sentido.

\section{Parque Natural Despeñaperros}

En el PORN del parque no se hace ninguna referencia específica, desde un punto de vista territorial, a como van a quedar reguladas ni desarrolladas las actividades recreativas (dentro del marco del uso público) así, por ejemplo, en las zonas de protección de grado $A$, se establece que "las actividades ligadas al uso público se podrán realizar en términos compatibles con la elevada protección de estos espacios, en el resto de áreas de protección el uso turístico-recreativo está permitido".

En el Capítulo III, sección segunda del PRUG (art. 24 al 27), también se encuentra breves indicaciones a la regulación de actividades de uso público dentro del parque: 
- Art.24, artículo semejante al ya analizado en otros parques que hace referencia a las licencias y documentación que deberán portar los practicantes de determinadas actividades dentro del parque.

- Art.25, es meramente informativo de la promoción que se va a realizar en el espacio del excursionismo utilizando la red de caminos y pistas forestales.

- Art.27, el único que hace referencia estricta a la ordenación territorial con relación a una actividad, en este caso la de acampada, la cual queda prohibida en todo el parque fuera de las áreas señalizadas para tal.

Por tanto, la única referencia concreta es la prohibición de la acampada libre en todo el parque fuera de las instalaciones establecidas para ello.

3.2. Perspectiva general del tratamiento dado a la regulación ter ritorial de las actividades recreativas en los PORN y PRUG

Tras analizar los documentos de planificación de los diecinueve Parques Naturales andaluces de montaña, la primera conclusión obtenida es la escasa presencia en la normativa de la regulación territorial de actividades recreativas. Esto es importante remarcarlo debido a que los PORN son un documento básico para la planificación en general, ya que según la ley 4/89 de "Conservación de los espacios naturales y de la flora y fauna silvestres", sus disposiciones suponen "un límite para cualquier otro instrumento de ordenación territorial o física", por tanto, para llevar a cabo cualquier proceso de planificación ambiental o territorial, se convierte en una figura fundamental. De igual forma, las consideraciones recogidas en el PRUG serán muy relevantes, ya que este es el documento que desarrolla operativamente la estrategia de ordenación de los PORN, utilizando para ello una serie de Programas Básicos de Actuación (entre los que se encuentran los Programas de uso público).

En los PORN y PRUG de estos espacios no aparecen prácticamente indicaciones que sean de utilidad en la toma de decisiones relacionadas con la regulación de actividades recreativas en estos espacios protegidos. Junto a esto cabe insistir que la inexistencia de los Programas de uso público en la gran mayoría de Parques, impide el poder generar un uso y disfrute adecuado de los espacios en cuestión. 
Si comparamos ambos documentos el PRUG suele traer consigo un mayor número de referencias relacionadas con la regulación de estas prácticas que los PORN.

Las puntualizaciones realizadas por los PORN son muy genéricas, de todas ellas las que aparecen en un mayor número de parque son las que establecen la prohibición de realizar cualquier actividad recreativa en las zonas de máxima protección, siendo esta una apreciación bastante limitante que debería venir acompañada de un estudio de detalle que mostrara la elevada fragilidad de los ecosistemas donde se están prohibiendo estas prácticas, con esto se podría evitar el malestar de determinadas empresas, grupos y asociaciones deportivas, que acusan a la Administración Ambiental de llevar a cabo una política en ocasiones demasiado prohibitiva.

Junto con esta premisa, también un elevado número de parques van a prohibir la actividad cinegética en las áreas de máxima protección.

Además de la caza, otras de las actividades que más comúnmente se prohíben en los Planes de Ordenación, son las que hacen mención a la circulación de vehículos a motor en zonas de elevada protección y las que deniegan la pernocta o acampada libre.

Se debe considerar que estas tres actividades:caza, rutas en vehículos a motor y acampada libre, suelen contar con un potencial impacto ambiental bastante negativo (siempre que su práctica conlleve un elevado grado de irracionalidad) pudiendo llegar a afectar al medio natural muy negativamente en forma de procesos erosivos, incendios forestales, contaminación de aguas, etc. (en algunos Parques como Grazalema o Sierra Nevada, en una misma norma se prohíben en las áreas de mayor protección tanto los recorridos con vehículos a motor como la caza). Por esto la legislación es tan limitativa en esta línea, ya que se entiende que es mejor restringir que luego compadecerse.

Tras esta serie de normas recogidas por un mayor número de parques, el resto son minoritarias y según las características naturales de los espacios y el real o potencial uso recreativo que presenten así son las regulaciones establecidas, por ejemplo, en un parque de uso básicamente metropolitano como el Parque Natural Montes de Málaga, uno de los mayores impactos es el producido por la actividad de esparcimiento en el medio natural, 
por ello en su PORN aparece una norma que prohíbe que dicho recreo concentrado se localice en cualquier espacio y sólo lo permite en las áreas recreativas establecidas para ello.

Junto con estas normas mencionadas, aparecen otras recogidas por los planes de un único parque, por tanto, quitando cierta generalización con relación a la regulación de las actividades cinegéticas, acampada y rutas en vehículos todo terreno, el resto de normas son muy diversas (atendiendo a los requerimientos de cada uno), pero sobre todo son escasas al considerar que el PORN es el documento básico de ordenación de los espacios protegidos y sus indicaciones se encuentran por encima de cualquier otra figura de planificación.

Con respecto a las referencias por parques, aquellos que recogen un mayor número de normas que regulan estas prácticas, son los de Sierra de las Nieves y Montes de Málaga, seguidos por los de Sierra Nevada y Sierra de Huétor.Por el contrario, los PORN de otra serie de espacios no hacen mención alguna en este sentido: Parque Natural Sierra Norte, Sierra de Aracena y Picos de Aroche, Despeñaperros, Sierras Subbéticas y Sierra Mágina, dejando libertad a las prácticas sin imponer ningún tipo de limitación.

Comparados con los PORN, en los PRUG aparecen un mayor número de referencias respecto a la regulación de las actividades.

De la normativa analizada, es la relacionada con la prohibición de la acampada libre dentro de los Parques (limitándola a los espacios establecidos para la misma), la que aparece referida en un mayor número de ocasiones. Junto con esta, la regulación sobre licencias y permisos que deben portar los practicantes de determinadas actividades es la siguiente en referirse por parte de un mayor número de planes.

Los impactos que pueden llegar a generar el tránsito de personas o vehículos fuera de las áreas establecidas para ello, hace que casi un $40 \%$ de los Planes Rectores de los Parques posean un artículo que sirva para regular los mismos.

Los problemas que pueden causar en el medio natural las actividades náuticas y aeronáuticas realizadas inconvenientemente (molestias a la fauna, contaminación de las aguas, etc.) condicionan que alrededor de un $22 \%$ de los parques recojan indicaciones en su PRUG, sin embargo, de nuevo hay que apuntar que estas limitaciones y prohibiciones debieran venir acom- 
pañadas por un estudio exhaustivo del medio que permitiesen prohibir o limitar con un criterio de protección avalado por un estudio científico.

En los Planes de Ordenación, los recorridos con vehículos a motor eran atendidos en la normativa de una serie de parques al poder llegar a ser una actividad ciertamente impactante y estresante para el medio, de igual modo, en los Planes Rectores de algunos espacios se recogen consideraciones en esta línea que prohíben las carreras con vehículos a motor, motocicletas y motocross y los recorridos con vehículos a motor en las zonas de máxima protección (Parque Natural de los Alcornocales, Sierra de Andújar y Sierra Mágina).

El resto de indicaciones ya se encuentran relacionadas con las características de los ecosistemas de cada uno de los parques, por ejemplo, en espacios que cuentan con una importante colonia de aves rapaces (Parque Natural de Sierra de Cazorla, Alcornocales o Grazalema), se limitan actividades que puedan llegar a afectarlas negativamente (escalada, actividades aéreas, etc.).

La mayor parte de los PRUG de los Parques Naturales andaluces en relación con los criterios de ordenación para actividades recreativo-deportivas en la naturaleza poseen semejanzas y diferencias:

- En general la mayor parte de las normas prestan atención a la circulación en los parques con los vehículos a motor y, normalmente, se establecen fuertes limitaciones (muy rígidas en el caso de pruebas deportivas). Donde se autoriza esta circulación se suelen establecer límites de velocidad, de tamaño de grupos, etc.

- Se suelen establecer requisitos distintos en función de si las prácticas son realizadas por personas federadas o no.

- Aparece prohibida la acampada libre exigiéndose la autorización de la Consejería de Medio Ambiente para obtener algún permiso.

- En relación con el excursionismo se suelen establecer itinerarios fijos debidamente señalizados con limitaciones en ocasiones (según la demanda, la fragilidad del medio o la estación). Los Planes Rectores de los Parques Naturales de los Montes de Málaga y Alcornocales, son los que presentan un mayor número de normas que regulan las prácticas recreativas en estos espacios protegidos andaluces, seguidos por los de 
Cazorla, Sierra de las Nieves, Sierra María-Los Vélez y Sierra de Andújar.Por el contrario, aquellos espacios que no presentan en sus documentos de planificación ninguna regulación en esta línea son los de Sierra de Aracena y Sierras Subbéticas, siendo ésta además prácticamente inexistente en el de Despeñaperros.

Las referencias del Parque Natural de Grazalema se han tomado de su Programa de uso público, de ahí que este sea el que presente la normativa más completa en este sentido (el resto de Parques deberían contar con este documento para que la regulación de las prácticas de uso público fuese lo más adecuada posible).

Los Parques que poseen una regulación más seria en esta línea junto con el de Grazalema, son el de los Montes de Málaga, Sierra de las Nieves, Alcornocales y Sierra María-Los Vélez. Por el contrario, aquellos que, o no atienden a ningún tipo de regulación en este sentido o la misma es muy escasa, son los de Sierra de Aracena y Sierras Subbéticas, que no poseen ningún tipo de normativa, por lo que la permisividad se impone en todas las prácticas con los problemas que esto puede llegar a generar, sobre todo considerando que en ambos parques existen empresas y asociaciones que están practicando actividades en contacto con la naturaleza: actividades aéreas, rutas a cabaIlo, ciclomontañismo, senderismo, etc. Junto con estos espacios, el Parque de Sierra Tejeda, Almijara y Alhama (que aún carece de PRUG) y el de Despeñaperros, también cuentan con una normativa prácticamente inexistente.

En otros espacios (Parque Natural de las Sierras de Cardeña y Montoro, Sierra de Hornachuelos, Sierra de Baza o Sierra Mágina) aunque la normativa es un tanto más completa, muchos de los artículos sólo se refieren a la limitación de acampada y recorridos en vehículos a motor y a la regulación de permisos y licencias que deben portar los practicantes de algunas actividades, con lo prácticamente no aparecen referencias en relación con la regulación territorial de las prácticas.

Las actividades que cuentan con una regulación mayor son: acampada, rutas en vehículos todo terreno y caza, frente a otras donde la normativa es muy escasa (espeleología) o inexistente (barranquismo).

Se puede afirmar que las figuras de planificación ambiental de los Parques Naturales andaluces de montaña contemplan, 
por lo general, escasamente la regulación de las actividades recreativas dentro de los mismos. Las normas que aparecen normalmente no van acompañadas de estudios de detalle del medio, que faciliten la toma de decisiones racionales en el proceso de planificación. Por esto la normativa que aparece es excesivamente prohibitiva en algunos casos y en otros demasiado permisiva.

Junto a esto, gran parte de los artículos son muy superfluos, genéricos y prácticamente semejantes en distintos Parques (por ejemplo, el que hace referencia a la regulación de permisos para la práctica de la actividad).

Concluyendo, el análisis de las figuras de planificación de los Parques Naturales de montaña de nuestra comunidad, muestra una muy pobre regulación territorial de las actividades de uso público (recreativas), los documentos de planificación ambiental no las atienden convenientemente ya que, un reglamento efectivo no supone limitar y prohibir los usos a realizar dentro de este espacio, sino que debe prever el disfrute de dichos recursos por parte de la población y compatibilizar la conservación del medio con la explotación racional de estos recursos. Ante esto se demanda una adecuada planificación de las mismas con las que se puedan evitar tanto problemas de degradación ambiental como de prohibiciones sin sentido.

\section{Referencias bibliográficas}

AGENCIA DE MEDIO AMBIENTE (1988): Plan director de uso y protección del Parque Natural de Grazalema. Junta de Andalucía. Sevilla.

BENAYAS DEL ÁLAMO, J. (coord.) (2000): Manual de buenas prácticas del monitor de naturaleza: espacios naturales pro tegidos de Andalucía. Consejería de Medio Ambiente, Junta de Andalucía, Sevilla.

CONSEJERÍA DE MEDIO AMBIENTE (1995): Plan de Ordenación de recursos naturales y Plan Rector de Uso y Gestión del Parque Natural Sierra de Aracena y Picos de Aroche. Junta de Andalucía. Sevilla.

CONSEJERÍA DE MEDIO AMBIENTE (1995): Plan de Ordenación de recursos naturales y Plan Rector de Uso y 
Gestión del Parque Natural Sierra Nevada. Junta de Andalucía. Sevilla.

CONSEJERÍA DE MEDIO AMBIENTE (1996): Plan de Ordenación de recursos naturales y Plan Rector de Uso y Gestión del Parque Natural Sierra María-Los Vélez. Junta de Andalucía. Sevilla.

CONSEJERÍA DE MEDIO AMBIENTE (1996): Plan de Ordenación de recursos naturales y Plan Rector de Uso y Gestión del Parque Natural Sierra Norte de Sevilla. Junta de Andalucía. Sevilla.

CONSEJERÍA DE MEDIO AMBIENTE (1996): Plan de Ordenación de recursos naturales y Plan Rector de Uso y Gestión del Parque Natural Sierras de Cardeña y Montoro. Junta de Andalucía. Sevilla.

CONSEJERÍA DE MEDIO AMBIENTE (1996): Plan de Ordenación de recursos naturales y Plan Rector de Uso y Gestión del Parque Natural Sierra de las Nieves. Junta de Andalucía. Sevilla.

CONSEJERÍA DE MEDIO AMBIENTE (1997): Plan de Ordenación de recursos naturales y Plan Rector de Uso y Gestión del Parque Natural Los Alcornocales. Junta de Andalucía. Sevilla.

CONSEJERÍA DE MEDIO AMBIENTE (1997): Plan de Ordenación de recursos naturales y Plan Rector de Uso y Gestión del Parque Natural Montes de Málaga. Junta de Andalucía. Sevilla.

CONSEJERÍA DE MEDIO AMBIENTE (1997): Plan de Ordenación de recursos naturales y Plan Rector de Uso y Gestión del Parque Natural de Despeñaperros. Junta de Andalucía. Sevilla.

CONSEJERÍA DE MEDIO AMBIENTE (1997): Plan de Ordenación de recursos naturales y Plan Rector de Uso y Gestión del Parque Natural Sierra de Hornachuelos. Junta de Andalucía. Sevilla.

CONSEJERÍA DE MEDIO AMBIENTE (1997): Plan de Ordenación de recursos naturales y Plan Rector de Uso y Gestión del Parque Natural Sierra de Castril. Junta de Andalucía. Sevilla.

CONSEJERÍA DE MEDIO AMBIENTE (1997): Plan de Ordenación de recursos naturales y Plan Rector de Uso y 
Gestión del Parque Natural Sierra de Andújar. Junta de Andalucía. Sevilla.

CONSEJERÍA DE MEDIO AMBIENTE (1997): Plan de Ordenación de recursos naturales y Plan Rector de Uso y Gestión del Parque Natural Sierra Subbética. Junta de Andalucía. Sevilla.

CONSEJERÍA DE MEDIO AMBIENTE (1997): Plan de Ordenación de recursos naturales y Plan Rector de Uso y Gestión del Parque Natural Sierra de Baza. Junta de Andalucía. Sevilla.

CONSEJERÍA DE MEDIO AMBIENTE (1997): Plan de Ordenación de recursos naturales y Plan Rector de Uso y Gestión del Parque Natural Sierra de Huétor. Junta de Andalucía. Sevilla.

Decreto 63/1994 de 15 de marzo por el que se aprueba el Plan de Ordenación de los Recursos Naturales del Parque Natural de Sierra Mágina.

Decreto 145/1999, de 15 de Junio por el que se aprueba el Plan de Ordenación de los Recursos Naturales del Parque Natural de las Sierras de Tejeda, Almijara y Alhama. BOJA n.․131. Sevilla, 11-11-1999.

Decreto 227/1999, de 15 de noviembre por el que se aprueba el Plan de Ordenación de los Recursos Naturales y el Plan Rector de Uso y Gestión del Parque Natural Sierra de Cazorla, Segura y las Villas. BOJA n. ${ }^{-149}$. Sevilla, 23-12-1999.

Ley 4/1989, de 25 de marzo, de Conservación de los Espacios Naturales y de la Flora y Fauna Silvestres.

MULERO MENDIGORRI, A. (2001): "Los espacios naturales protegidos en Andalucía: evolución, caracterización geográfica y singularidades", Ería, 54-55. Departamento de Geografía, Universidad de Oviedo, Oviedo, pp. 161-179. 